\title{
Machine Learning Approach for National Innovation Performance Data Analysis
}

\author{
Dominik Forner ${ }^{1} \mathbb{1}^{\mathrm{a}}$, Sercan Ozcan ${ }^{1} \mathbb{1}^{\mathrm{b}}$ and David Bacon $^{2} \mathbb{C}^{\mathrm{c}}$ \\ ${ }^{1}$ Faculty of Business and Law, University of Portsmouth, Portland Street, Portsmouth, U.K. \\ ${ }^{2}$ Institute of Cosmology and Gravitation, University of Portsmouth, Burnaby Road, Portsmouth, U.K.
}

Keywords: Innovation Performance, Neural Networks, Machine Learning, National Innovation System, Economic Growth, Innovation Policy, Decision Support System.

\begin{abstract}
National innovation performance is essential for being economically competitive. The key determinants for its increase or decrease and the impact of governmental decisions or policy instruments are still not clear. Recent approaches are either limited due to qualitatively selected features or due to a small database with few observations. The aim of this paper is to propose a suitable machine learning approach for national innovation performance data analysis. We use clustering and correlation analysis, Bayesian Neural Network with Local Interpretable Model-Agnostic Explanations and BreakDown for decomposing innovation output prediction. Our results show, that the machine learning approach is appropriate to benchmark national innovation profiles, to identify key determinants on a cluster as well as on a national level whilst considering correlating features and long term effects and the impact of changes in innovation input (e.g. by governmental decision or innovation policy) on innovation output can be predicted and herewith the increase or decrease of national innovation performance.
\end{abstract}

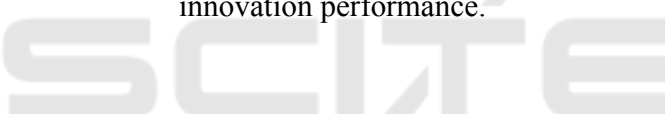

\section{INTRODUCTION}

In the new global economy, national innovation performance has become a central issue for being economically competitive (Izsak et al., 2013). It has therefore been the subject of much systemic investigation. Whilst traditional innovation system research focused in the past decades on the qualitative analysis of activities and relations of different actors within an innovation system, recent approaches apply machine learning techniques for a quantitative approach. Nevertheless, they are either limited due to qualitatively selected features or due to a small database with few observations. In any case, it is not possible to predict the effect of changing key determinants on innovation output and the associated impacts on national innovation performance in the innovation ecosystem. This study therefore proposes a suitable machine learning approach for national innovation performance data analysis.

\section{LITERATURE}

The following section outlines two themes of literature, national innovation performance including systems of innovation as well as machine learning approaches in recent studies in the field of innovation management.

\subsection{National Innovation Performance}

All systems of innovation approaches, starting with National Systems of Innovation (NIS) (Freeman, 1987; Dosi, 1988) contribute to a general understanding of innovation (Lundvall, 2004) and the interrelationships of a variety of different elements in innovation systems (Izsak et al., 2013), but the boundaries between the systems become blurred (Leydesdorff, 2000).

Different organisations and institutions developed innovation indexes. An innovation index constitutes the different facets of a NIS (Crespo and Crespo,

\footnotetext{
https://orcid.org/0000-0001-7100-4998

b(D) https://orcid.org/0000-0002-0482-7529

(1D https://orcid.org/0000-0002-2562-8537
} 
2016) and facilitates the evaluation of innovation capability (Wang and Gong, 2017). Since it is difficult to determine an overall outcome of policy instruments, an innovation index also measures the effectiveness of government intervention (Mahroum and Al-Saleh, 2013).

Current trends in the research of innovation systems have led to a proliferation of studies that use modern analytical methods to identify specific determinants in innovation indexes and to predict the effect of policy instruments.

\subsection{Machine Learning Approaches}

Data Envelopment Analysis (DEA), stochastic frontier analysis (SFA) (Liu et al., 2018) and Fuzzy Set Quantitative Comparative Analysis (fsQCA) are currently the most popular methods for investigating innovation performance quantitatively. One of the main obstacles is that studies are limited in terms of selecting the relevant features and with regard to the number of observations used for calculation (Cooper and Glaesser, 2011; Kane et al., 2014). Furthermore, QCA performs incorrect for causal inference and its application should be discontinued (Baumgartner and Thiem, 2017).

Recent studies therefore use machine learning techniques to overcome these limitations. Hajek and Henriques (2017) demonstrated in their study, that the importance of determinants of regional innovation performance can be identified using multi-output neural networks. However, the determinants used for analysis have been evaluated empirically based on two community innovation surveys instead of applying unsupervised feature selection.

Pençe, Kalkan and ÇeSmeli (2018) followed a similar approach by applying artificial neural network on a Global Innovation Index (GII) data set of 2016. They identified 27 features that increase innovation performance. However, the study is limited due to the observation of one single year and not considering long-term effects and correlations.

One study of Minniti and Venturini (2017) predicted the growth effect of policy instruments targeting business R\&D by using a novel regression procedure on industry data from the United States.

\subsection{Literature Gap and Objective}

This section has attempted to provide a brief summary of the literature relating to national innovation performance, innovation index based research and application of machine learning techniques. The studies reviewed here consistently indicate that a suitable approach for the analysis of national innovation performance using machine learning techniques has not yet been found. Current approaches are either limited due to qualitatively selected features or due to a small database with few observations. In any case, it is not possible to predict the effect of changing key determinants on innovation output and the associated impacts on national innovation performance in the innovation ecosystem.

Therefore, the aim of this paper is to propose a suitable machine learning approach for national innovation performance data analysis.

\section{METHODOLOGY}

This study follows the ongoing trend in innovation index based research. Firstly, an appropriate innovation index is selected followed by data collection and preparation. Secondly, interrelations of innovation performance have been investigated and national innovation profiles have been established. In a third step, key determinants have been identified and their effect on national innovation performance has been analysed. In a fourth step, the prediction of innovation output is decomposed and the importance of features as well as their effect in case of change is investigated. Finally, conclusions were drawn for the development of a decision support system for innovation policy in consideration of prior results. The analysis is performed using R ( $\mathrm{R}$ Core Team, 2017) and Python (van Rossum and Drake, 2011). The terms 'feature' and 'determinant' are used interchangeably in this study.

\subsection{Data Collection and Preparation}

Different innovation indexes and scoreboards have been assessed and the most appropriate index has been selected according to the following criteria.

- Published yearly

- Main focus on innovation performance

- Assessment on national level

- Index data is accessible and comparable

- Data used by indexes is retrieved from reliable sources

All criteria are met by the GII. It is constituted by an input / output framework with 7 pillars and 21 categories, containing 81 indicators of which 57 are related to hard data, 19 are composite indicators and 5 are related to survey questions whereas data is retrieved from 30 different reliable sources. Data of 
the GII has been retrieved from its web source ${ }^{4}$ covering a five years' time frame from 2014 to 2018 . The data files have been consolidated by mapping features column-wise and countries row-wise. Each row represents the country, year of observation and the values related to the 81 features.

Subsequently the data set has been cleaned by removing countries without full records. The resulting data set contains 120 countries with 480 observations. Missing values of some countries have been imputed using multiple imputation by chained equations (MICE) with the predictive mean matching method of the caret package (Kuhn, 2017). Herewith, values are predicted as close as possible in relation to observed values, whereas the distribution resembles that from ordinal logistic regression.

\subsection{Understanding Interrelationships and Establishing National Innovation Profiles}

Understanding the interrelations and establishing national innovation profiles was achieved by applying cluster analysis, correlation analysis, time series analysis and principal component analysis (PCA).

Clustering the data set has been performed using Clustering for Large Applications (CLARA) with the 'Manhattan' metric according to Kaufman and Rousseeuw (1990) of the cluster package (Maechler et al., 2017). The chosen metric is less sensitive concerning outliers by calculating the distances as a sum of absolute differences. CLARA thereby clusters only a sample of the full data set and assigns subsequently all objects to the respective clusters. The number of clusters has been determined with the gap statistic method (Tibshirani and Hastie, 2006).

Following the descriptive analysis of the interrelationships between countries in terms of innovation performance, the interrelationships of the individual features were analysed by means of correlation analysis. Thereby, any values between 0.1 and -0.1 have been declared as not correlating.

A time series analysis was conducted to investigate if long-term effects of features exist. Therefore, the data set has been converted into a time series object using the xts package (Ryan and Ulrich, 2017) and is analysed further using autocorrelation function (ACF) of the stats package ( $\mathrm{R}$ Core Team, 2017).

Establishing national innovation profiles has been performed using PCA and investigating the component loadings. PCA transforms the data to a new set of uncorrelated variables, where the first components represent the most variance of all principal components (Joliffe, 2002). The features of a national innovation profile in the original feature space are mapped on the same component.

Results are visualised using the ggplot2 package (Wickham, 2016) for PCA, the factoextra package (Kassambara and Mundt, 2017) for CLARA, the seaborn package (Waskom et al., 2018) for the correlation heatmap in python and the zoo package (Zeileis and Grothendieck, 2005) for the ACF plot.

\subsection{Identifying Key Determinants and Investigating Their Effect on National Innovation Performance}

Different machine learning models have been cross validated using the train function in the caret package (Kuhn, 2017), inter alia tree-based models, boosting models and neural network models. The method used for cross validation was $\mathrm{k}$-fold cross-validation whereas $\mathrm{k}=10$. The data set was divided into learning and testing in a three-quarter ratio. The decisive criterion for the model selection was the Root Mean Square Error (RMSE) value.

Model performance plots, learning curves and residuals diagnostic plots were created with the DALEX package (Biecek, 2018) and analysed to evaluate the performance of the model, goodness of fit and to audit residuals.

In order to investigate the effects of key determinants on national innovation performance, the functioning of the model is analysed. Results of Merging Path Plot based on Tukey HSD test are compared to CLARA results to identify countries with similar factorisation related to innovation output in the ecosystem. In addition, global variable importance has been assessed overall and on a cluster level to specify the key determinants. Their effect on innovation output has been investigated with Partial Dependency Plots.

\subsection{Decomposing Innovation Output Prediction}

Prediction of innovation output is decomposed with breakdown plot (Staniak and Biecek, 2018) to investigate the attribution of key determinants. Further, their importance has been assessed by Local Interpretable Model-Agnostic Explanations (Pedersen and Benesty, 2018). To assess whether and

\footnotetext{
${ }^{4}$ https://www.globalinnovationindex.org/analysis-indicator
} 
how changes in key determinants (e.g. by innovation policy and governmental decisions) are reflected in innovation output, ceteris paribus plots (Biecek, 2019) are created and interpreted.

\subsection{Establishing a Decision Support System for Innovation Policy}

In consideration of the results of the descriptive analysis of the interrelations in the innovation ecosystem, the national innovation profiles and the effect of identified key factors on innovation performance, conclusions were drawn for the development of a decision support system for innovation policy.

\section{RESULTS}

This section outlines the results of the descriptive analysis for establishing national innovation profiles, investigating correlation as well as long-term effects of features and the identification of national innovation performance key determinants using Bayesian Neural Network as well as decomposing the prediction for innovation output.

\subsection{Clustering, Correlation and Time Series Analysis}

The result of CLARA clustering has been visualised with two dimensions, explaining about $45.6 \%$ of point variability (see Figure 1). Countries are positioned in the visual in relation to their innovation performance in the innovation ecosystem. Thereby, clusters can be labelled as Innovation Performer, Innovation Follower, Innovation Challenger, and Underdeveloped. Interestingly, some clusters are overlapping and some countries managed to shift to a higher cluster in some years, others did not manage to improve whilst being in an overlapping area.

Also, the position of China has to be highlighted, which is in the Innovation Follower cluster and distanced from the other countries. Further investigation of these cases revealed, that countries with features of the category innovation diffusors in their national innovation profile managed to shift into a higher cluster. This category contains features related to business sophistication, education, infrastructure, political and regulatory environment.

China has a very diversified innovation profile with dynamically changing composition of features. There is a lack of innovation diffusor features and the profile is mainly constituted by creative outputs, market sophistication and knowledge diffusion.

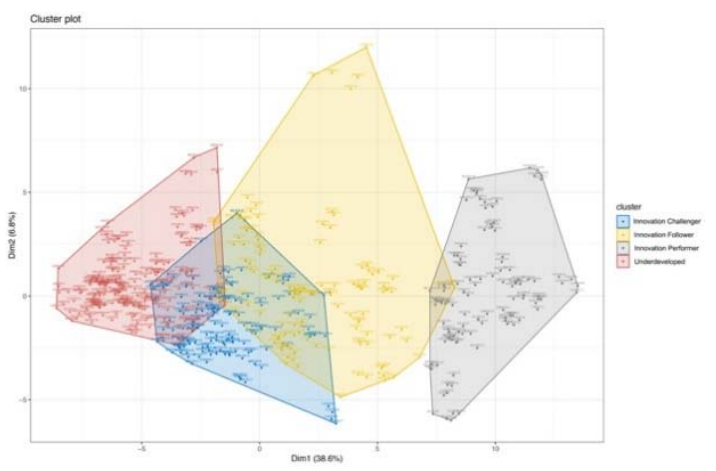

Figure 1: CLARA Cluster Plot of Countries.

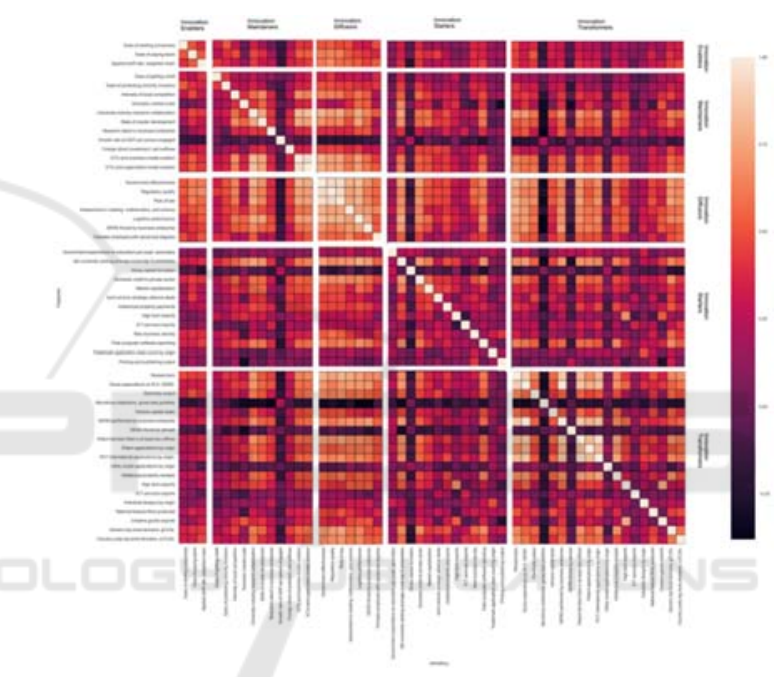

Figure 2: Correlation Matrix of Clusters and Features.

Looking at the correlation matrix of clusters and features (see Figure 2), positive as well as negative correlations have been identified. There was a significant positive correlation between researchers and gross expenditure on R\&D (0.89) and between researchers and GERD performed by business enterprise (0.86). Another significant positive correlation has been identified between Gross expenditure on R\&D and GERD performed by business enterprise (0.98) and patent families filed in at least two offices (0.86). The most significant negative correlation has been found between high tech imports and ICT services imports $(-0.78)$.

Figure 3 shows the time series plot of features whereby the feature index is on the $\mathrm{x}$-axis and the mean values on the $y$-axis. Lines are plotted for the years 2014-2017. Looking at features between the index 1-10 (1), outliers mainly exist in 2017 and 2014 , indicated by a lower value. 


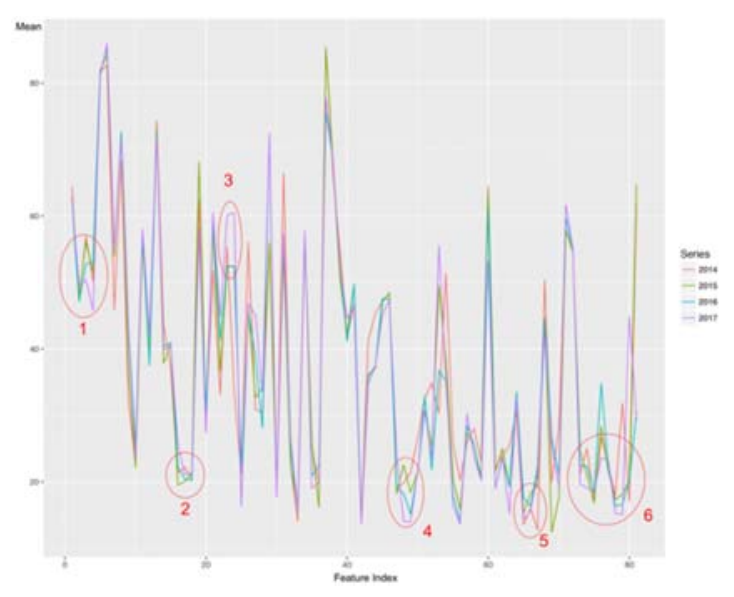

Figure 3: Time Series Plot of Features.

Furthermore, it appears that features in 2016 2017 are changing regarding their values compared to 2014-2015. This change can also be seen between features 11-20 (2), additionally values in 2014 show an upward trend whilst in other years a downward trend. For features between 41-50 (4) this contrary trend can also be observed, 2016-2017 going down whilst 2014-2015 going up, being the same between features 61-70 (5) and features 71-81 (6). At some features (3) a shift can be observed as well for 20162017 with higher values than features of 2014-2015. In general, it can therefore be stated that some features are affected prior to 2016, which leads to a change for the following years. Furthermore, time lags have been identified in the ACF-plot that occure within two years from or prior to the point of observation.

\subsection{Model Validation, Identification of Key Determinants and Decomposing Prediction}

The cross-validation of machine learning (ML) methods revealed that Bayesian Regularized Neural Network (BRNN) fits according to its RMSE value better than Boosted Smoothing Splines (bstSm), Random Forests (rf) and Extreme Gradient Boosting Trees (xgbTree) in relation to the GII dataset (see Figure 4). Further, the learning curve of BRNN is with regard to resampling, testing and training data almost congruent from a training size of 200 (see Figure 5). As a result of the cross-validation of ML models, Bayesian Regularized Neural Network has been selected for further analysis due to its performance and goodness of fit.

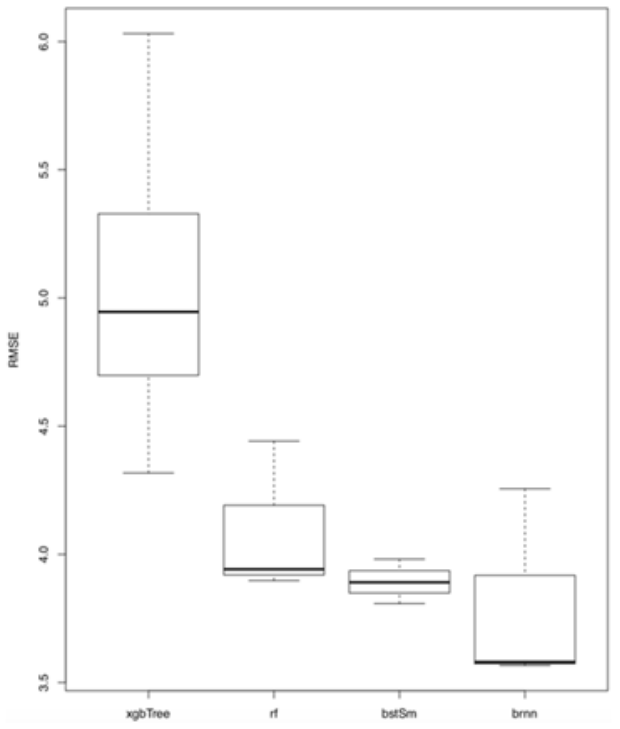

Figure 4: Cross-Validation of Machine Learning Models.

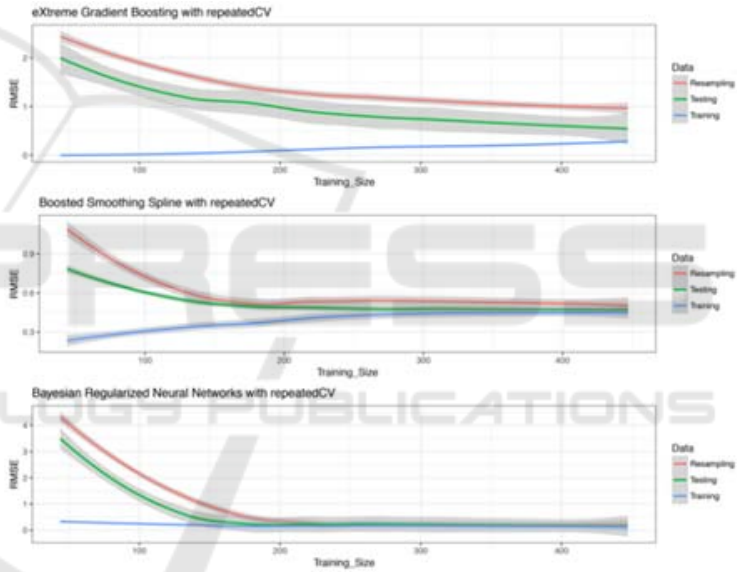

Figure 5: Learning Curves of Machine Learning Models.

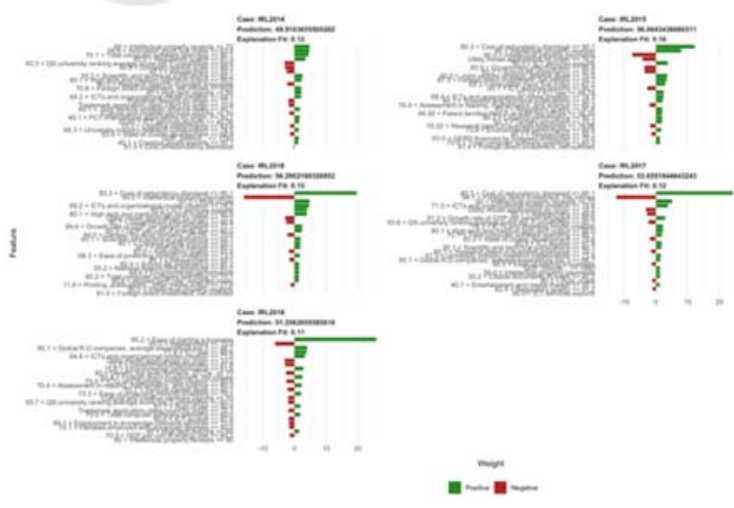

Figure 6: Explanations for Ireland 2014 - 2018.

Proxy variables have been identified for countries with similar profiles and predictions have been 
decomposed. As one result the proxy variables 'Cost of redundancy dismissal' and 'Scientific and technical publications' are notable, which have both a strong positive impact on the top countries' innovation performance (CHE, SWE, NLD, GBR, SGP). In Figure 6, the explanations between 2014 and 2018 are exemplarily illustrated. Therefore, key determinants can be identified that have a positive and negative effect on innovation output. Comparing the national feature importance to the global feature importance, intellectual property receipts is most important for this cluster in 2018 whereas it has a negative impact for Ireland. Moreover, ease of starting a business is most important for Irelands' innovation output in 2018. Indicators reported by innovation indexes can therefore not easily be taken for decision making. Moreover, key determinants for innovation performance need to be assessed on national level. Besides the feature importance, the attribution of this feature to innovation output in general has been assessed by decomposing the prediction. Changes in innovation input effect to the percentage of it attribution the innovation output and herewith increases or decreases national innovation performance.

\section{CONCLUSIONS}

Overall, these results indicate that the machine learning approach is appropriate to benchmark national innovation profiles, to identify key determinants on a cluster as well as on a national level whilst considering correlating features and long term effects and the impact of changes in innovation input (e.g. by governmental decision or innovation policy) on innovation output can be predicted and herewith the national innovation performance increase or decrease.

\section{REFERENCES}

Baumgartner, M. and Thiem, A. (2017) 'Often Trusted but Never (Properly) Tested: Evaluating Qualitative Comparative Analysis', Sociological Methods and Research, pp. 1-33.

Biecek, P. (2018) 'DALEX: explainers for complex predictive models', ArXiv e-prints. Available at: https://arxiv.org/abs/1806.08915.

Biecek, P. (2019) 'ceterisParibus: Ceteris Paribus Profiles'. Available https://cran.rproject.org/package $=$ ceterisParibus.

Cooper, B. and Glaesser, J. (2011) 'Paradoxes and pitfalls in using fuzzy set QCA: Illustrations from a critical review of a study of educational inequality', Sociological Research Online. Durham University, 16(3).

Crespo, N. F. and Crespo, C. F. (2016) 'Global innovation index: Moving beyond the absolute value of ranking with a fuzzy-set analysis', Journal of Business Research, 69(11), pp. 5265-5271.

Dosi, G. (1988) Technical change and economic theory. International Federation of Institutes for Advanced Study Research Series, no. 6.

Freeman, C. (1987) Technology policy and economic performance: lessons from Japan, London: Pinter. Pinter Pub Ltd.

Hajek, P. and Henriques, R. (2017) 'Modelling innovation performance of European regions using multi-output neural networks.', PLOS ONE. Public Library of Science, 12(10), pp. 1-21.

Hajek, P., Henriques, R. and Hajkova, V. (2014) 'Visualising components of regional innovation systems using self-organizing maps-Evidence from European regions', Technological Forecasting and Social Change, 84.

Izsak, K., Markianidou, P. and Radošević, S. (2013) 'Lessons from a Decade of Innovation Policy - What can be learnt from the INNO Policy TrendChart and The Innovation Union Scoreboard', Final Report, European Union, pp. 1-103.

Joliffe, I. T. (2002) Principal Component, Principal Component Analysis SE - 7. New York: Springer (Springer series in statistics).

Kane, H. et al. (2014) 'Using qualitative comparative analysis to understand and quantify translation and implementation', Translation Behavioral Medicine. Germany: SPRINGER SCIENCE AND BUSINESS MEDIA, (2), p. 201.

Kassambara, A. and Mundt, F. (2017) 'factoextra: Extract and Visualize the Results of Multivariate Data Analyses'. Available at: https://cran.rproject.org/package $=$ factoextra.

Kaufman, L. and Rousseeuw, P. J. (1990) 'Clustering Large Applications (Program CLARA).', Finding Groups in Data: An Introduction to Cluster Analysis, p. 126.

Kuhn, M. (2017) 'caret: Classification and Regression Training'. Available at: https://cran.rproject.org $/$ package $=$ caret.

Leydesdorff, L. (2000) 'The triple helix: An evolutionary model of innovations', Research Policy, 29(2), pp. 243-255.

Liu, Z. et al. (2018) 'Industrial development environment and innovation efficiency of high-tech industry: analysis based on the framework of innovation systems', Technology Analysis and Strategic Management, 30(4), pp. 434-446.

Lundvall, B.-Å. (2004) 'National Innovation Systems Analytical Concept and Development Tool', DRUID Tenth Anniversary Summer Conference 2005. Australia: CARFAX PUBLISHING TAYLOR \& FRANCIS LTD, (1), p. 43.

Maechler, M. et al. (2017) 'cluster: Cluster Analysis Basics and Extensions'. 
Mahroum, S. and Al-Saleh, Y. (2013) 'Towards a functional framework for measuring national innovation efficacy', Technovation. Elsevier, 33(1011), pp. 320-332.

Minniti, A. and Venturini, F. (2017) 'The long-run growth effects of R\&D policy', Research Policy. Elsevier B.V., 46, pp. 316-326.

Pedersen, T. L. and Benesty, M. (2018) 'lime: Local Interpretable Model-Agnostic Explanations'. Available at: https://cran.r-project.org/package=lime.

Pençe, I., Kalkan, A. and Çeşmeli, M. Ş. (2018) ‘Estimation of the Country Ranking Scores on the Global Innovation Index 2016 Using the Artificial Neural Network Method', International Journal of Innovation and Technology Management. Bucak Zeliha Tolunay Applied Technology and Business School, Mehmet Akif Ersoy University, Adem Tolunay Campus: World Scientific Publishing Co. Pte Ltd, p. 1940007.

R Core Team (2017) 'R: A Language and Environment for Statistical Computing'. Vienna, Austria. Available at: https://www.r-project.org/.

van Rossum, G. and Drake, F. L. (2011) The Python Language Reference Manual. Network Theory Ltd.

Ryan, J. A. and Ulrich, J. M. (2017) 'xts: eXtensible Time Series'. Available at: https://cran.rproject.org $/$ package $=$ xts.

Staniak, M. and Biecek, P. (2018) 'Explanations of Model Predictions with \{live\} and \{breakDown\} Packages', ArXiv e-prints. Available at: https://arxiv.org/abs/1804.01955.

Tibshirani, R. and Hastie, T. (2006) 'Estimating the number of clusters in a data set via the gap statistics', Journal of the Royal Statistical society. Blackwell Publishers, 63(2), pp. 411-423.

Wang, H. and Gong, S. (2017) 'Research on the Innovation Evaluation Indexes for Innovation-Oriented City', DEStech Transactions on Economics and Management, (iceme-ebm).

Waskom, M. et al. (2018) 'mwaskom/seaborn: v0.9.0 (July 2018)'. doi: 10.5281 /zenodo. 1313201 .

Wickham, H. (2016) ggplot2: Elegant Graphics for Data Analysis. Springer-Verlag New York. Available at: http://ggplot2.org.

Zeileis, A. and Grothendieck, G. (2005) 'zoo: S3 Infrastructure for Regular and Irregular Time Series', Journal of Statistical Software, 14(6), pp. 1-27. 